\title{
Primary school-based food environment intervention for improved behaviour, food knowledge and dietary habits: results from Project Daire, a randomised-controlled, factorial design cluster trial evaluation
}

\author{
Sarah F. Brennan ${ }^{1,2}$, Fiona Lavelle ${ }^{1}$, Sarah E. Moore ${ }^{1,2}$, Moira Dean ${ }^{1}$, \\ Michelle C. McKinley ${ }^{1,2,4}$, Patrick McCole ${ }^{3}$, Ruth F. Hunter ${ }^{1}$, Laura Dunne ${ }_{1}^{4}$, \\ Niamh E. O'Connell ${ }^{1}$, Chris R. Cardwell ${ }^{2}$, Chris T. Elliott ${ }^{1}$, Danielle McCarthy ${ }^{1}$ and \\ Jayne V. Woodside ${ }^{1,2,4}$ \\ ${ }^{1}$ Institute for Global Food Security, Queen's University Belfast, Belfast BT9 5 AG, \\ ${ }^{2}$ Centre for Public Health, Queen's University Belfast, Belfast, BT12 6BA, \\ ${ }^{3}$ Queen's Management School, Queen's University Belfast, Belfast, BT9 5EE and \\ ${ }^{4}$ Centre for Evidence and Social Innovation, Queen's University Belfast, Belfast, UK
}

Evidence suggests that dietary intake of UK children is suboptimal ${ }^{(1)}$. It is therefore imperative to identify effective and sustainable methods of improving dietary habits and food knowledge in this population. Schools are recognised as ideal environments for public health interventions and the effectiveness of a 'whole-school' approach has been highlighted ${ }^{(2,3)}$. Project Daire, in partnership with schools and a range of stakeholders, aimed to improve children's health-related quality of life, emotional and behavioural wellbeing, food knowledge and dietary habits via two multi-component interventions.

Daire was a randomised-controlled, factorial design trial evaluating two interventions. Primary schools in the North West of Northern Ireland were recruited and randomised to one of four 6-month intervention arms: i) 'Nourish', ii) 'Engage', iii) 'Nourish' and 'Engage' and iv) Delayed. 'Nourish' was an intervention aiming to alter the whole-school food environment, incorporate food-related experiences and increase exposure to local foods. 'Engage' was an age-appropriate, cross-curricular educational intervention on food, agriculture, food and nutrition-related sciences and associated careers. Primary outcomes were emotional and behavioural wellbeing measured using the Strengths and Difficulties Questionnaire and health-related quality of life measured using the KIDSCREEN-10 questionnaire, and they are presented alongside a number of secondary outcomes including dietary intake, perceived cooking competence and food knowledge measures.

A total of $903(n=445$ aged 6-7 years and $n=458$ aged $10-11$ years $)$ primary school pupils participated in the trial. Emotional and behavioural wellbeing, measured via Total Difficulties Score, improved in all pupils who received the 'Nourish' intervention compared with those that did not (adjusted difference in mean $=-0.82 ; 95 \% \mathrm{CI}-1.45,-0.17 ; \mathrm{P}<0.02$ ). No significant difference in Health-Related Quality of Life was observed between groups. The 'Nourish' intervention also produced some changes in school-based dietary behaviour, most apparent in the 10-11 year old pupils, whilst understanding of food labels (adjusted difference in mean $=0.15$; $95 \% \mathrm{CI}$ : $0.05,0.25 ; \mathrm{P}<0.01$ ) seasonality of vegetables (adjusted difference in mean $=0.29 ; 95 \% \mathrm{CI}$ : $0.01,0.56$; $\mathrm{P}=0.04$ ), openness to trying new foods (adjusted difference in mean $=0.27 ; 95 \% \mathrm{CI}: 0.03,0.51 ; \mathrm{P}=0.03$ ) and cooking competence improved (adjusted difference in mean $=3.21 ; 95 \% \mathrm{CI}: 0.65,5.77 ; \mathrm{P}=0.02)$ when compared to pupils who did not receive the 'Nourish' intervention. In $10-11$ yearold pupils who received the 'Engage' intervention compared with those who did not, their understanding of food labels significantly improved (adjusted difference in mean $=0.70 ; 95 \% \mathrm{CI}: 0.30,1.10 ; \mathrm{P}<0.01$ ).

Improvements in childhood emotional and behavioural wellbeing, dietary intake, knowledge about food, cooking skills and willingness to try new foods were more strongly associated with the 'Nourish' whole-school food environment intervention in the participating schools. Exploration of the sustainability and long-term effectiveness of such whole-school food interventions should be investigated to further explore their impact and feasibility across regions.

1. NatCen Social Research, MRC Elsie Widdowson Laboratory (2018) Data Collection SN: 6533.

2. Weichselbaum, E., Buttriss, J.L. (2014) Nutr Bull 39, 9-73.

3. Wang, D., Stewart D (2012) Public Health Nutrition 16, 1082-100. 